This report was prepared as an account of work sponsored by an agency of the United States Government. Neither the United States Government nor any agency thereof, nor any of their employees, makes any warranty, express or implied, or assumes any legal liability or responsibility for the accuracy, completeness, or usefulness of any information, apparatus, product, or process disclosed, or represents that its use would not infringe privately owned rights. Reference herein to any specific commercial product, process, or service by trade name, trademark, manufacturer, or otherwise does not necessarily constitute or imply its endorsement, recommendation, or favoring by the United States Government or any agency thereof. The views and opinions of authors expressed herein do not necessarily state or reflect those of the

United States Government or any agency thereof.
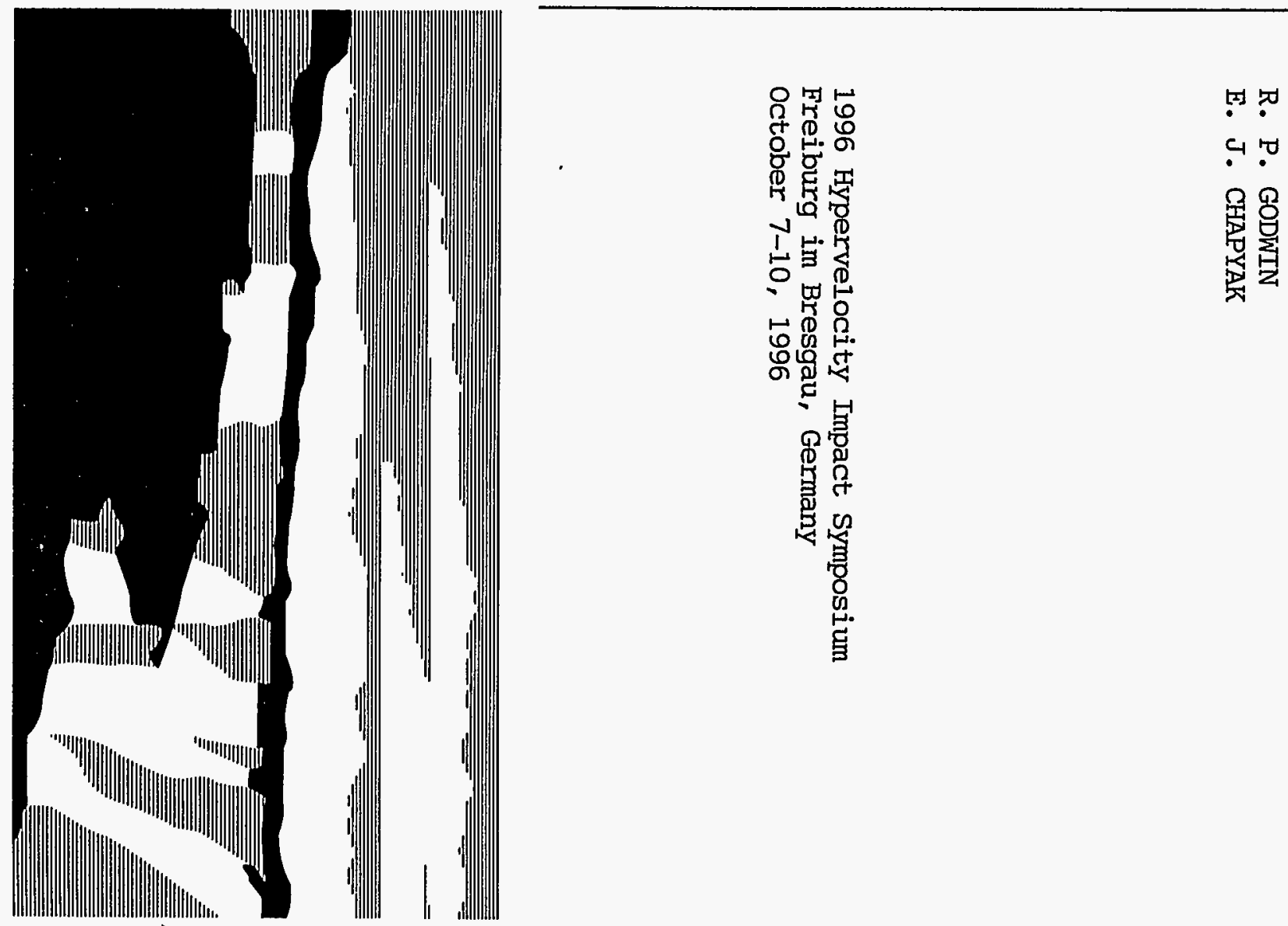


\section{DISCLAIMER}

Portions of this document may be illegible in electronic image products. Images are produced from the best available original document. 


\title{
APPARENT TARGET STRENGTH IN LONG-ROD PENETRATION
}

\author{
R. P. GODWIN and E. J. CHAPYAK \\ Applied Theoretical and Computational Physics Division \\ Group XNH, MS F664 \\ Los Alamos National Laboratory \\ Los Alamos, New Mexico 87545
}

\begin{abstract}
We investigate the apparent enhancement of target strength in the steadystate Tate model of long-rod penetration. We show that computing the effective area over which the target behaves as a fluid provides an explanation of the effective 1-D target strength measured empirically. Expressing the effective target strength as $R_{t}=a \cdot Y_{t}$, we postulate that $a=A_{c} / A_{p}$, where $Y_{t}$ is the nominal strength; $A_{c}$ is the effective target fluid cross-sectional area and $A_{p}$ the projectile cross-sectional area. For the case of a rod and projectile of the same material, we use the Tate model together with the jet model of Birkhoff $e t$ $a l$. to show $a \approx 4$ is likely. Simultaneously satisfying Newton's Second Law and the Tate model yields a very general derivation of $a=4$. By explicitly including strength terms in both the Tate equation and Newton's Second Law, an even more general $a=f\left(v, \rho_{p}, \rho_{t}, Y_{p}, Y_{t}\right)$ can be derived.
\end{abstract}

\section{INTRODUCTION}

Assuming the penetrator and target behave as ideal incompressible fluids provides an excellent approximation for the penetration of metal jets or longrods into semi-infinite targets. In steady-state flow, Bernoulli's Law gives pressure balance on the penetrator axial streamline at the penetrator-target interface with

$$
\frac{1}{2} \rho_{p}(v-u)^{2}=\frac{1}{2} \rho_{t} u^{2}
$$

The penetrator is incident with velocity $v$ and penetrates with velocity $u ; \rho_{p}$ and $\rho_{t}$ are the projectile and target densities. This is the essence of the Tate model (Tate 1967, Tate 1969, and Walters 1989). If the penetrator length to diameter ratio, $L / D$, is large, the effects of the pressure spike at the initial penetrator-target impact ("primary penetration") and of inertial effects after the penetrator is consumed ("secondary penetration") can be neglected. The steady-state condition of Eq. (1.1) is satisfied if

$$
u=v /(1+\mu) \text {, }
$$

where $\mu \equiv \sqrt{\rho_{t} / \rho_{p}}$. Equation (1.1) predicts a total penetratio

$$
P=L u /(v-u)=L / \mu \text {. }
$$


Equations (1.1)--(1.3) are surprisingly successful in predicting long-rod and jet penetration. Attempts to improve this fluid model by including material strength corrections are empirically successful. However, they have generally been weakly justified conceptually or justified with complex arguments. This is unfortunate considering the elegant simplicity and success of the Tate model in its purely fluid form. Our goal is to explain the effective Tate model target strength, while maintaining the essential simplicity of the model.

Letting $Y_{p}$ and $R_{t}$ be appropriate strengths for the projectile and target, we generalize Eq. (1.1) to

$$
\frac{1}{2} \rho_{p}(v-u)^{2}+Y_{p}=\frac{1}{2} \rho_{t} u^{2}+R_{t} .
$$

If $Y_{p}$ and $R_{t}$ are equal in magnitude, their effects cancel. A naive model predicts no strength effects with a rod projectile and target of the same material, if $Y_{p}=R_{t}$. Choosing $Y_{p}$ and $R_{t}$ empirically to fit rod penetration data for projectiles and targets of the same nominal strength $Y_{o}$, yields $Y_{p} \sim Y_{o}$ and $R_{t} \sim 4 Y_{0}$. The effective enhancement of the target strength has been attributed to strain hardening and the unusual nature of the stress at the yield site, but has more recently been recognized as due primarily to geometrical effects (Tate 1967, Walters 1989, and Hohler 1990). According to Anderson and Walker (1991), " $R_{t}$ embodies both the compressive strength of the target as well as the need to account for target confinement in a one-dimensional context. Hence, $R_{t}$ incorporates the effect of the extent and rate of plastic deformation in the target." We will show that 2-D hydrodynamic effects are sufficient to explain the effective enhanced target strength of the Tate model.

\section{THE ROD PENETRATOR AS AN INVERSE BIRKHOFF JET}

Since Bernoulli's equation is a function only of the squares of velocities, it is invariant under time reversal. We therefore refer to the classic paper on jet formation by Birkhoff et al. (1948) for guidance. We have shown numerically that the assumption of invariance under time reversal is a good approximation even for realistic material equations of state with strong shocks present (Godwin 1992a,b). In 2-D cartesian coordinates, a jet formed by a collapsing "cone" (i.e., wedge) of half angle $\beta$ satisfies the following relations

and

$$
\frac{m_{j}}{m_{s}}=\frac{(1-\cos \beta)}{(1+\cos \beta)} \equiv \tan ^{2} \frac{\beta}{2}
$$

$$
\frac{V_{j}}{V_{s}}=\frac{(1+\cos \beta)}{(1-\cos \beta)}
$$

(Walters 1989). This analytic solution in cartesian coordinates qualitatively describes the behavior of cylindrical explosively-formed jets. We assume, therefore, that it will also qualitatively describe our cylindrical problem. For our inverse jet (i. e., rod penetration) problem, we associate $m_{j} \leftrightarrow m_{p}$, where $m_{j}$ is the mass per unit length of the jet and $m_{p}$ the mass per unit length of the 
long-rod penetrator. Similarly, $m_{s} \leftrightarrow m_{t}$, where $m_{s}$ is the mass per unit length of the slug and $m_{t}$ the mass per unit length of that portion of the penetrator target which is behaving as a fluid. Determining $m_{t}$ will provide as estimate of the area over which the target behaves as a fluid or, equivalently, the area over which the target strength is effective. We further associate $V_{j} \leftrightarrow v$ and $V_{s} \leftrightarrow 0 ; V_{j}$ is the jet velocity, $v$ the penetrator velocity, $V_{s}$ the slug velocity, and $u$ the rod-penetration velocity. In this application of the Birkhoff jet model, the velocity of the "effective slug" in the laboratory frame of reference is $V_{s} \equiv 0$. From Eqs. (2.1) and (2.2),

$$
\beta=2 \cdot \arctan \sqrt{\left(V_{s} / V_{j}\right)}=2 \cdot \arctan \sqrt{0}=0^{\circ}
$$

and the flow is precisely parallel to the symmetry axis. This is an important result and quite different from the usual jet situation where $\beta \sim 20^{\circ}$ and the slug velocity, $V_{s}$, is a few percent of the jet velocity.

For the case of a rod and target of the same material, Eq. (1.2) requires $u / v=m_{p} / m_{t}=1 / 2$ and $P=L$ for consistency between the Tate and Birkhoff models. Since we have assumed a penetrator and target of the same density,

$$
\frac{m_{t}}{m_{p}}=2=\frac{\rho_{t} A_{t}}{\rho_{p} A_{p}}=\frac{A_{t}}{A_{p}}=\frac{T_{t}}{T_{p}}
$$

$T_{l} / T_{p}=2$ is the ratio of the target to projectile thickness in this 2-D cartesian model. We assume that, in the similar cylindrical case, $a=\left(T_{t} / T_{p}\right)^{2}=4$.

\section{NEWTON'S SECOND LAW}

We apply Newton's Second Law

$$
\frac{d(m v)}{d t}=v \frac{d m}{d t}+m \frac{d v}{d t}=F
$$

to the hydrodynamic steady-state penetration of an infinitely long rod into a semi-infinite target. We also assume that the counter flow is anti-parallel to the rod, as justified in Sec. 2. The projectile mass, $m$, decreases as the rod erodes, but $v$ (the velocity of the rear of the rod) is constant. In the laboratory reference frame, where the rod material is moving; the incoming portion at $v$ and, consistent with the application of Bernoulli's Law and with the Birkhoff model of Sec. 2, an axial back flow portion at $(v-2 u)$. Since, in this steady-state model, $d v / d t \equiv 0$, Eq. (3.1) applied to all rod material gives

$$
\begin{aligned}
\frac{d(m v)}{d t}= & \left.\frac{d(m v)}{d t}\right|_{\leftarrow}+\left.\frac{d(m v)}{d t}\right|_{\rightarrow}= \\
& v \rho_{p} A_{p}(v-u)+(v-2 u) \rho_{p} A_{p}(v-u)=2 \rho_{p} A_{p}(v-u)^{2} .
\end{aligned}
$$

The arrows indicate forward and backward axial flow. According to Bernoulli's Law, the force at the erosion front is

$$
F=p A_{e}=\frac{1}{2} \rho_{p}(v-u)^{2} \cdot A_{c} \text {. }
$$


The effective area of the erosion front is $A_{e}$ and not equal to $A_{p}$. Define $a \equiv A_{e} / A_{p}$ as before. We find $a \equiv 4$, an important result derived solely from Bernoulli's Law and Newton's Second Law. It agrees with the estimate of Sec. 2 based on the Birkhoff jet model, but is much more general.*

We predict a discontinuous increase in the flow cross-sectional area at the point where the rod material velocity drops from $v$ to $u$. This increase in area is evident in the capped steady-state penetration jet observed in radiographs (Hohler 1990) and in hydrodynamic simulations (Anderson 1993). The increase in area is closely related to the linear hydraulic bore and radial hydraulic jump (Godwin 1993). Such discontinuities are the incompressible flow analogs of shocks in compressive flow (Landau 1959).

*If one neglects the second term of Eq. (3.2) or if $u=v / 2$, Newton's law gives

$$
a^{*}=\frac{2}{1-u / v}=\frac{2}{1-(1+\mu)^{-1}}=2+\frac{2}{\mu} \text {. }
$$

This is equivalent to ignoring the counter streaming target material or to assuming there is no slip at the interface of the static target material and the counter streaming projectile material. We suspect, that in many physical situations, the existence of slip at the interface of the counter-flowing projectile material and the target material breaks down. In the noslip case, Eq. (3.4) may be a good approximation to the flow dynamics. For the case of a projectile and target of the same material $u=v / 2, \mu=1$, and there is no slip. In this case, $a^{*}=a=4$.

\section{4. " $1 \frac{1}{2}-D$ COMPLETE" TATE MODEL}

The elegantly simple Tate fluid model of long-rod penetration has been usefully applied for nearly 30 years. The enhanced effective target strength required to fit experimental data has not been complemented by a straightforward analytical model. We have shown that the effective strength is a result of the spatial extent of fluid behavior and that Newton's Second Law gives a selfconsistent " $1 \frac{1}{2}-\mathrm{D}$ complete" Tate model. The experimentally measured $R_{t} \approx 4 Y_{t}$ is suggested by our model. The model can be straightforwardly extended to explicitly include the effects of material strength.

\section{ACKNOWLEDGMENT}

This Los Alamos National Laboratory research was supported by the US DoD Ballistic Missile Defense Office. 


\section{REFERENCES}

Anderson, C. E. Jr., D. L. Littlefield, and J. D. Walker (1993). Long-Rod Penetration, Target Resistance, and Hypervelocity Impact. Int. J. Impact Engng., 14, 1.

Anderson, C. E. Jr. and J. D. Walker (1991). An Examination of Long-Rod Penetration. Int. J. Impact Engng., 11(4), 481.

Birkhoff, G., D. MacDougall, E. Pugh, and G. Taylor (1948). Explosives with Lined Cavities. J. Appl. Phys., 19(6), 563.

Birkhoff, G. (1950). Chap. II. Hydrodynamics, A Study in Logic, Fact, and Similitude (Princeton Press, Dover reprint 1955).

Godwin, R. P. (1992a). Simulations of Birkhoff Jets. Los Alamos National Laboratory. Memorandum X-3:92-282U, unpublished.

Godwin, R. P. (1992b). Inverse Simulations of Birkhoff Jets. Los Alamos National Laboratory. Memorandum X-3:92-283U, unpublished.

Godwin, R. P. (1993). The Hydraulic Jump ("shocks" and viscous flow in the kitchen sink). Am. J. Phys., 61(9), 829.

Landau, L. D. and E. M. Lifshitz (1959). Fluid Mechanics (Pergamon Press), 396.

Hohler, V. and Stilp, A. J. (1990). Long-Rod Penetration Mechanics. Chap. 5 of High Velocity Impact Dynamics, ed. J. A. Zukas (John Wiley \& Sons).

Tate, A. (1967). A Theory for the Deceleration of Long Rods After Impact. J. Mech. Phys. Solids, 15, 387.

Tate, A. (1969). Further Results in the Theory of Long Rod Penetration. J. Mech. Phys. Solids. 17, 141.

Walters, W. P. and J. A. Zukas (1989). Chap. 5, Shaped Charge Jet Formation, Fundamentals of Shaped Charges (John Wiley \& Sons) . 